\title{
Heartbeat Detection Using Three-Axial Seismocardiogram Acquired by Mobile Phone
}

\author{
F Landreani ${ }^{1}$, D Golier ${ }^{2}$, A Hossein ${ }^{2}$, J Rabineau ${ }^{2}$, P van de Borne ${ }^{3}$, E Caiani ${ }^{1}$, P-F Migeotte ${ }^{2}$ \\ ${ }^{1}$ Dipartimento di Elettronica, Informazione e Bioingegneria, Politecnico di Milano, Milano, Italy \\ ${ }^{2}$ Laboratory of Physics and Physiology, Université Libre de Bruxelles, Bruxelles, Belgium \\ ${ }^{3}$ Department of Cardiology, Hopital Erasme, Université Libre de Bruxelles, Bruxelles, Belgium
}

\begin{abstract}
Recent studies have demonstrated the possibility to acquire the heartbeat induced vibrations resembling the seismocardiogram (SCG) by the mobile phone accelerometer (m-SCG). As most of ECG-free beat detection algorithms are based on processing one m-SCG component only (e.g. head-to-foot), our aim was to propose an alternative and robust method to extract beatto-beat systolic complex (SC) from three-axial m-SCG and test its occurrence compared to R peak of ECG.

Eleven male subjects (mean age 22 \pm 3 ) were recruited and $\mathrm{m}$-SCG signal $(50 \mathrm{~Hz})$ was acquired for 5 minutes by a smartphone positioned on the chest.

Newtonian equations of kinematics and continuous wavelet transform were applied to the m-SCG signal to detect beat-by-beat SC occurrence.

Data from one subject were discarded due to low quality signal (feasibility 91\%). High values of sensitivity (0.995), positive predictive value (0.974) and accuracy (0.96) were obtained. The explored heart rate range (45$101 \mathrm{bpm})$ was quite wide and acceptable limits of agreement $( \pm 1- \pm 6$ bpm) were achieved.
\end{abstract}

\section{Introduction}

The seismocardiogram (SCG) represents the localized vibrations of the ribcage induced by the heart activity and blood shifted through the aortic tree to the vascular periphery at each cardiac cycle. This signal can be acquired by mobile phone accelerometers and it is known as mobileSCG (m-SCG).

A sequence of sharp deflections characterizes the SCG morphology; these waves usually are associated to specific cardiac mechanic events such as the isovolumetric contraction (IVC) and aortic opening (AO) [1]. These sharp deflections could be considered as a systolic complex (SC), for simplicity, given that their occurrence indicate the onset of the systolic phase.

Most of ECG-free beat detection algorithms process only one m-SCG component. The most common way to proceed in the SC detection is to consider the dorso-ventral component. In particular, the detection of the IVC and AO deflections on the m-SCG using only one component is sometimes complex: the annotation of $\mathrm{m}-\mathrm{SCG}$ is quite complex given that the waveform amplitudes can be weakened or amplified by the smartphone position on the body surface or by the body shape or posture during the acquisition and by the breathing.

Different ECG-independent techniques have been applied to detect the fiducial peaks mainly considering only one axis of the smartphone's accelerometer [2]. These algorithms work on strong assumptions (time or amplitude thresholds) on the waveform complex that could lead to misdetection and then to erroneous beat-to-beat heart rate estimation. The amplitude of SC is mainly influenced by the inter- and intra-subject variability, which could lead these algorithms to erroneous fiducial peaks detection.

However, as in the m-SCG, three components are available thanks to the triaxial, useful information could be accessible by using also the other two components (headto-foot and right-to-left directions. In this way, the threedimensional nature of the heartbeat-induced vibrations can be fully taken into account.

In order to overcome these limitations, our aim was to propose an ECG-free method to extract beat-to-beat systolic complex, considering all the 3-axial accelerometric components of the m-SCG signal, based on the Newtonian equations of kinematics already implemented by Migeotte et al. in precedent researches $[3,4]$. The proposed method is based on the calculation of the signal power and on the continuous wavelet transform. 


\section{Materials and Methods}

\subsection{Experimental set-up and protocol}

The protocol here described was conducted in accordance to the ethics defined in the Helsinki Declaration of 1975, as revised in 2000. Eleven healthy subjects were recruited (mean age: $22 \pm 3$ ) after the Ethical approval and signed consent.

The m-SCG signals were recorded using a smartphone application (iPhone $6, \mathrm{fs}_{\mathrm{m}-\mathrm{SCG}}=50 \mathrm{~Hz}$ ) placed at the sternum near the cardiac apex while subject was in supine position (Fig. 1).

One-lead electrocardiogram was recorded in parallel to m-SCG using the KINO device (fs KINO$=200 \mathrm{~Hz}$; developed by Laboratory of Physics and Physiology, LPHYS, Bruxelles).

The devices were manually synchronized. The subjects completed a five-minute controlled breathing (CB) protocol during which the ECG and m-SCG were simultaneously recorded. The beginning of the m-SCG recording matched the onset of the $\mathrm{CB}$ protocol.

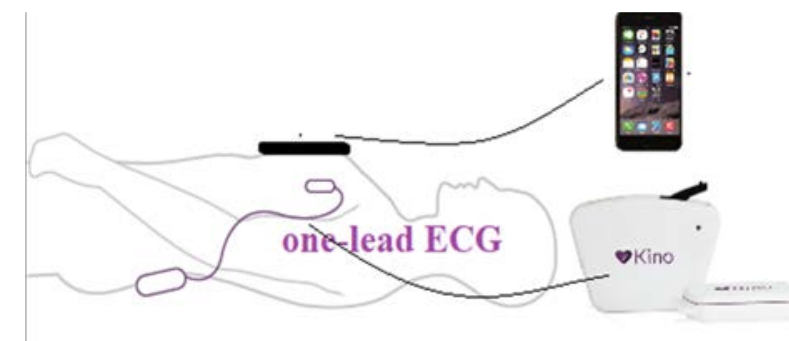

Figure 1 Schematic representation of the experimental set-up; the m-SCG was acquired by smartphone positioned on the sternum of the subject, while in supine posture, without any externally connected devices. Simultaneously, one lead ECG was acquired by Kino ${ }^{\circledR}$ device. The synchronization between the two devices was done manually.

\subsection{Beat-to-beat systolic complex detection}

The ECG and m-SCG were up sampled $(1000 \mathrm{~Hz})$. The ECG signals were processed using wavelet-based ECG delineator to derive the $\mathrm{R}$ peak positions from which the time series (RR), representing the heart cycle durations, were calculated and considered as gold standard.

The pre-processing step consisted in filtering the $\mathrm{m}$ SCG in order to remove the noise level. A Butterworth passband filter, which cut-off frequency were set at 10 and $180 \mathrm{~Hz}$, was applied to preserve the heartbeat sounds and to remove the baseline drift and motion-related artefacts, as shown in Fig. 2. On the Z-component of m-SCG, the systolic complex is shown and is identified with the sharp deflection composed by the IVC and AO waves (Fig. 2).

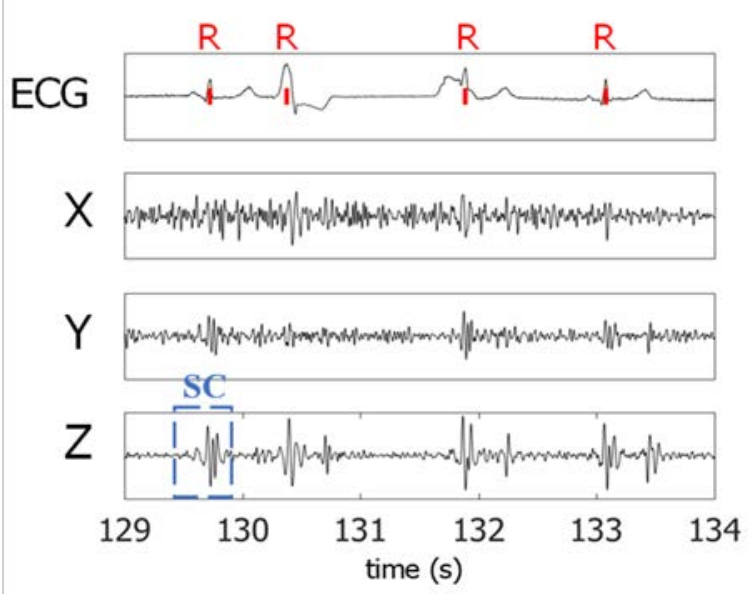

Figure 2 The three axial mobile seismocardiogram $(X, Y, Z)$ simultaneously with the ECG is shown with over imposed the $R$ peaks (red) and the systolic complex (SC, blue) which represent the heartbeat occurrence. The SC includes the IVC and AO waves; the SC amplitude depends on the physiological conditions of the subject (e.g. respiration pattern, age, body mass composition).

The procedure to detect the systolic complex is explained in these successive steps:

a. Calculation of the linear Power

The tree-axial seismocardiogram $(\mathrm{X}, \mathrm{Y}, \mathrm{Z})$ by the smartphone accelerometer was considered to calculate the Newtonian equations of kinematics. Considering the mass of the subject $(\mathrm{m})$, once calculated the acceleration $(\vec{a})$ and the velocity $(\vec{v})$ vectors, the method proceeds on the calculation of the linear power P (Fig 3) as:

$$
P=m \cdot \vec{a} \cdot \vec{v}
$$

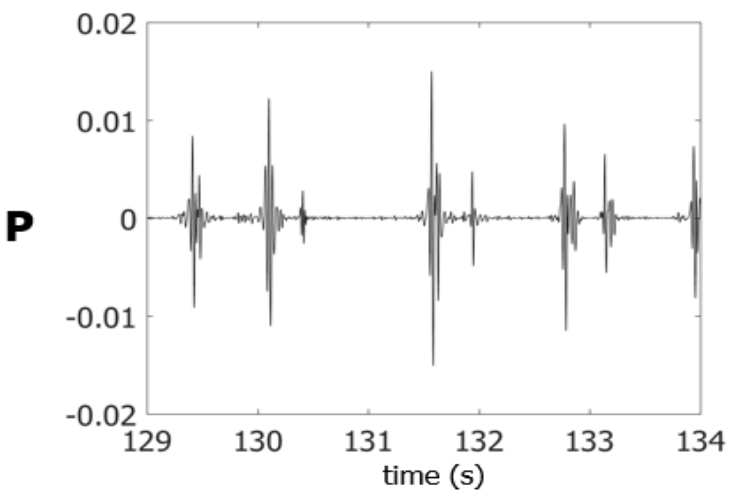

Figure 3. Example of the linear Power (P) calculated taking into account the tri-axial m-SCG. 
b. Quadratic spline decomposition

The decomposition was chosen because it provides a representation of the features of the signal at different resolutions. The linear Power is decomposed (Fig.4) as a set of functions up to $5^{\text {th }}$ order using the quadratic spline wavelet, which is implemented in the waveletbased ECG delineator[5]. As the SC shows a significant component at scale $2^{4}$; this component was thus considered for the continuous wavelet transform.

c. Continuous wavelet transform

Once applied the continuous wavelet transform, its signal amplitude was calculated; the maxima peaks were associated to the SCs that were detected using the local maxima research function (Fig. 5).

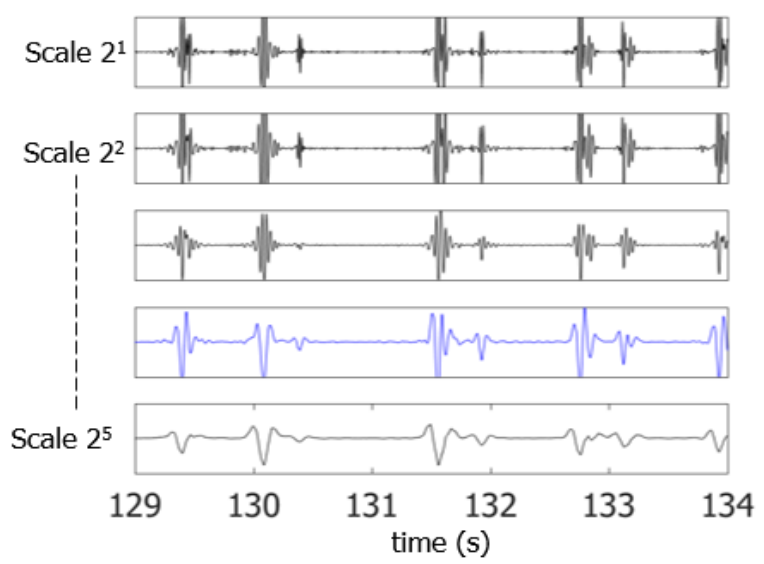

Figure 4 The quadratic spline decomposition of the linear power $\mathrm{P}$ is represented. The $2^{4}$ scale (blue) was considered for the systolic complexes' detection.

\section{d. Accuracy control}

The SC detected were visually inspected using as reference the $\mathrm{R}$ peak from the ECG. The SC complexes corresponding correctly to the $\mathrm{R}$ peaks were considered True Positives (TP), then the ones in the mistaken position or the double peaks were classified as False Positives (FP), then the missing detection were considered as False Negatives (FN). The performance of the proposed method for the SC detection using three-axial seismocardiogram was measured by sensitivity, positive predictivity and accuracy.

Once the correct complexes were defined, the time difference between successive peaks ( $\mathrm{R}$ and SC) was calculated and the time series were obtained (RR and SCSC, respectively) as shown in Fig, 6. The RR series were considered as the gold standard.
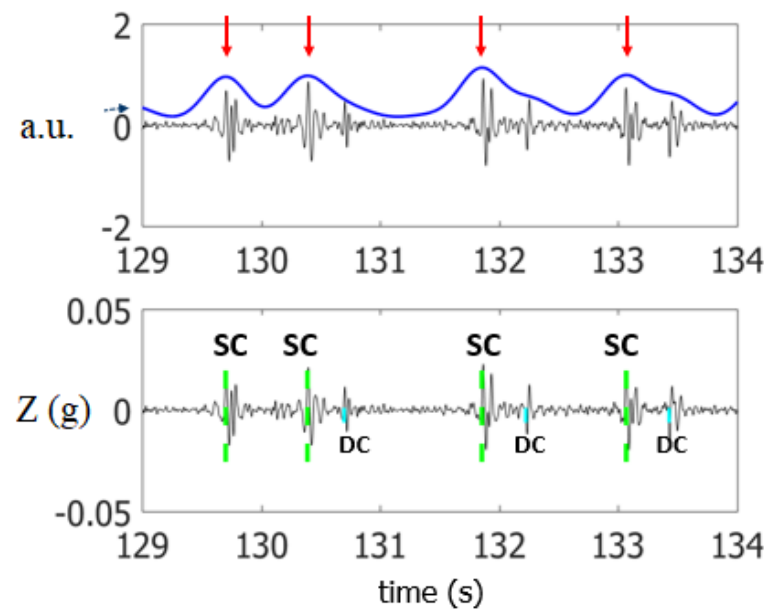

Figure 5. In the upper panel, on the continuous wavelet transform amplitude signal (blue) are indicated the local maxima (red arrows) corresponding to the systolic complexes; in the lower panel, the method proceeds in the detection of the SC (green dashed line) using the local maxima function. The algorithm is also able to detect the diastolic complexes (DC).
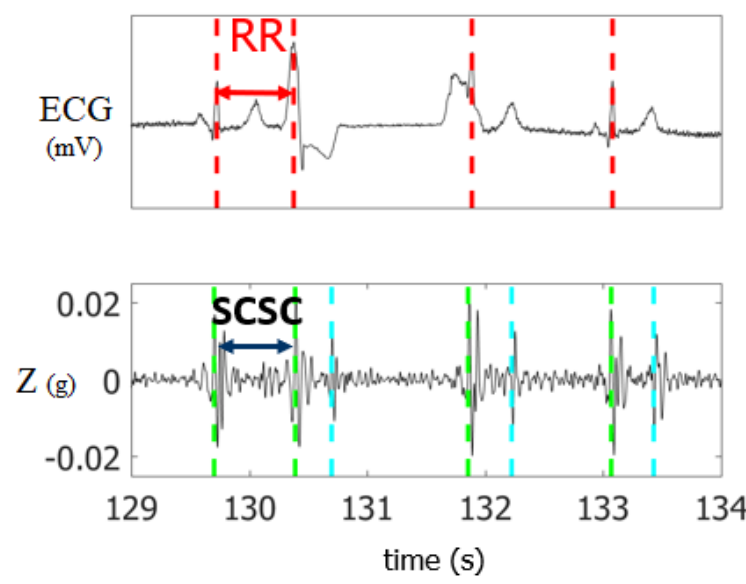

Figure 6. Time series calculated considering the $\mathrm{R}$ peaks and SC complexes (RR in red, SCSC in green dashed lines). The SCSC series were so compared to the gold standard.

\subsection{Validation and statistical analysis}

Linear correlation and Bland-Altman analyses were applied to test if the SCSC series obtained from three-axial m-SCG, could represent a validate surrogate of the heartbeat duration obtained from the ECG. The analysis consisted in comparing the SCSC series with the RR series extracted from ECG. 


\section{Results}

The signal from 10 subjects were processed, one was discarded for poor quality signal. A feasibility of $91 \%$ was obtained.

A total of 2816 beats were correctly identified (TP), 76 beats identified as FP, and 14 as FN. The performance of the method proposed obtained high values of sensitivity (0.995), positive predictivity (0.974) and accuracy (0.96).

High $\mathrm{R}^{2}$ value (0.989) and narrow confidence interval $(\mathrm{CI}= \pm 35 \mathrm{~ms}, \pm 2 \mathrm{SD})$ were obtained on all the range of $\mathrm{RR}$ duration of the experimental set (0.59-1.33 s), as shown in Fig 7.
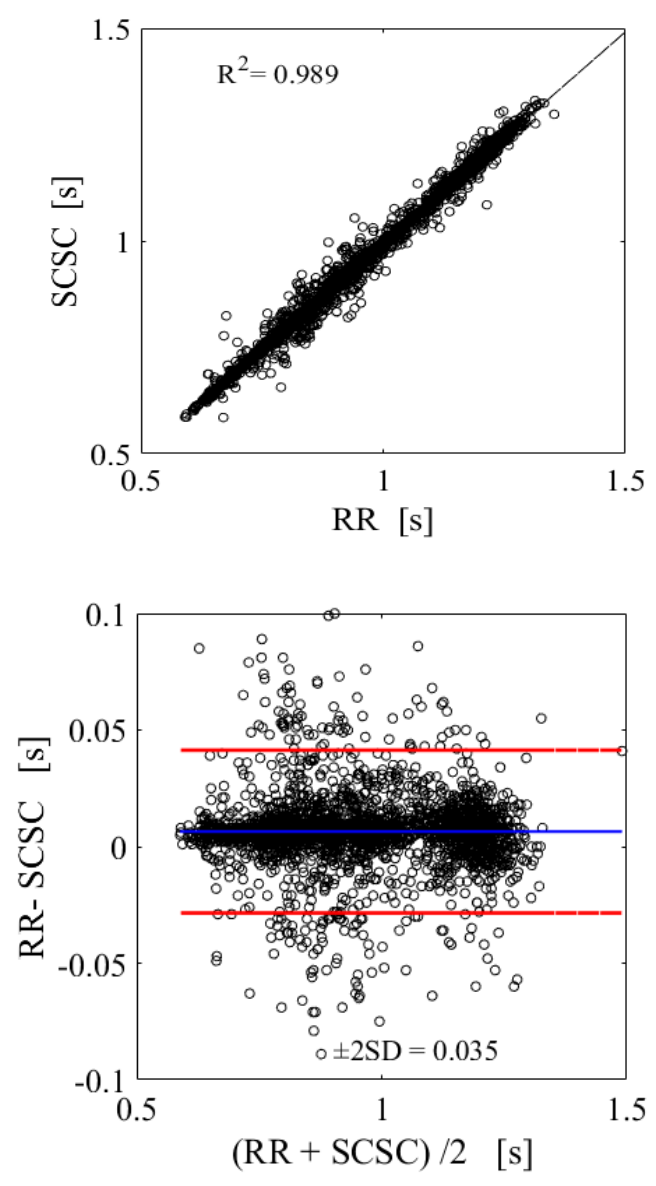

Figure 7. Linear correlation and Bland Altman analyses shows good correspondence between the RR and SCSC duration intervals and narrow limits of agreement.

\section{Conclusions}

It has been shown the feasibility of the proposed method for the detection of the heartbeat occurrences as systolic complex using the three-axial SCG and m-SCG.
The algorithm explored a quite wide range of heart rates (45-101 bpm) during a five minutes breathing protocol. Acceptable limits of agreement were obtained, $\pm 1 \mathrm{bpm}$ and $\pm 6 \mathrm{bpm}$ for the slowest and fastest heart rate, respectively.

The feasibility of the proposed method should be further studied using smartphone's measurements of subjects with different cardiovascular condition, especially those with abnormal respiratory sinus arrhythmia.

\section{Acknowledgements}

P.F. Migeotte is supported by the Belgian Federal Science Policy Office (BELSPO) via the ESA PRODEX program.

We acknowledge the support of the Italian Space Agency (contract 2013-064-R.0 "3D Ballistocardiography in microgravity”, PI EG Caiani)

\section{References}

[1] J. M. Zanetti, M. O. Poliac and R. S. Crow, "Seismocardiography: waveform identification and noise analysis," [1991] Proceedings Computers in Cardiology, Venice, Italy, 1991, pp. 49-52. doi: 10.1109/CIC.1991.169042

[2] F. Landreani \& E.G. Caiani (2017) Smartphone accelerometers for the detection of heart rate, Expert Review of Medical Devices, 14:12, 935-948, DOI: 10.1080/17434440.2017.1407647

[3] P.-F. Migeotte, J. Monfils, F. Landreani, I.I. Funtova, J. Tank, P. Van De Borne, E.G. Caiani; Cardiac strength deconditioning after the 60-days head-down bed-rest assessed by heart kinetic energy wearable monitoring, European Heart Journal, Volume 38, Issue suppl_1, 1 August 2017

[4] A. Hossein et al; Kino-Cardiography, A New Wearable Monitoring Technique: A Randomized Double-Blind Crossover Validation With Echocardiography; Journal of Hypertension. 36():e219, 2018, DOI: 10.1097/01.hjh.0000539620.28080.56 , Issn Print: 02636352

[5] J. P. Martinez, R. Almeida, S. Olmos, A. P. Rocha and P. Laguna, "A wavelet-based ECG delineator: evaluation on standard databases," in IEEE Transactions on Biomedical Engineering, vol. 51, no. 4, pp. 570-581, April 2004. doi: 10.1109/TBME.2003.821031

Address for correspondence.

Federica Landreani

DEIB - Piazza Leonardo da Vinci, 32

20133 Milano

Federica.landreani(at)polimi.it 